This document is the accepted manuscript version of the following article: Holler, M., Odstrci1, M., Guizar-Sicairos, M., Lebugle, M., Mü1ler, E., Finizio, S., ... Aeppli, G. (2019). Three-dimensional imaging of integrated circuits with macro- to nanoscale zoom. Nature Electronics.

https://doi.org/10.1038/s41928-019-0309-z

\title{
Three-dimensional imaging of integrated circuits with macro to nanoscale zoom
}

\author{
Mirko Holler ${ }^{1, *}$, Michal Odstrcil ${ }^{1}$, Manuel Guizar-Sicairos ${ }^{1}$, Maxime Lebugle ${ }^{1}$, \\ Elisabeth Müller ${ }^{1}$, Simone Finizio ${ }^{1}$, Gemma Tinti $^{1}$, Christian David ${ }^{1}$, Joshua Zusman², \\ Walter Unglaub2 ${ }^{2}$, Oliver Bunk ${ }^{1}$, Jörg Raabe ${ }^{1}$, A. F. J. Levi², Gabriel Aeppli ${ }^{1,3,4}$
}

${ }^{1}$ Paul Scherrer Institut, CH-5232 Villigen PSI, Switzerland.

2Department of Electrical and Computer Engineering, University of Southern California, Los Angeles, CA 90089-2533, USA.

${ }^{3}$ Department of Physics, ETH Zürich, CH-8093 Zürich, Switzerland.

${ }^{4}$ Institut de Physique, EPFL, CH-1015 Lausanne, Switzerland.

*Correspondence to: mirko.holler@psi.ch

The imaging of integrated circuits across different length scales is required for failure analysis, design validation and quality control. Currently such inspection is accomplished using a hierarchy of different probes, from optical microscopy on the millimetre length scale to electron microscopy on the nanometre scale. Here we show that ptychographic X-ray laminography (PyXL) can provide non-destructive, three-dimensional views of integrated circuits, yielding both images of an entire chip volume and high-resolution images of arbitrarily chosen sub regions. We demonstrate the approach using chips produced with $16 \mathrm{~nm}$ fin field-effect transistor technology, achieving a reconstruction resolution of $18.9 \mathrm{~nm}$, and compare our results to photolithographic mask layout files and more conventional imaging approaches such as scanning electron microscopy. The technique will also be applicable to other branches of science and engineering where three-dimensional X-ray images of planar samples are required.

Non-destructive three-dimensional imaging over large volumes with nanoscale resolution is important for a variety of applications, from mapping the natural neural connectome $e^{1,2}$ to inspecting silicon-based integrated circuits $3,4,5,6$. For integrated circuits, such inspection is required for failure analysis and security ${ }^{7}$, and a hierarchy of probes are currently used to image at various length scales: from imaging the entire chip (millimetres) using optical microscopy to imaging individual features of the underlying transistors (nanometres) using transmission electron microscopy on thin slices prepared with a focused ion beam.

X-ray ptychography combines scanning X-ray microscopy and coherent diffractive imaging ${ }^{8}$. A sample is scanned in a coherent X-ray beam and the propagated diffraction patterns are recorded, in reciprocal space, for each scanning position. Iterative algorithms permit the reconstruction of projections of the sample, while the resolution is limited neither by the beam diameter nor step size used in the scan, but is determined by the scattered X-rays that can be recorded having a sufficient signal-to-noise ratio as well as the position accuracy and stability of the sample in the beam. Recently, we showed that ptychographic X-ray computed tomography (PXCT) - the combination of X-ray ptychography and computed tomography, which involves recording many projections of a sample at different sample orientations - can provide high-resolution 3D images of a complementary metal-oxide-semiconductor (CMOS) integrated circuit at $14.6 \mathrm{~nm}$ isotropic resolution ${ }^{5}$.

In tomography, the axis of rotation is perpendicular to the propagation direction of the incident light. As a consequence, samples need to be specially created such that they can be accessed by the radiation from all projection angles from 0 to 180 degrees. For integrated circuits, this requires the extraction of a close to cylindrical pillar from the chip which, in our previous demonstration, was prepared by focused ion beam milling. While the subsequent tomography requires no slicing of the pillar, and is in that sense non-destructive, the extraction of the imaged pillar is a destructive and time-consuming task. Additionally, the region to be imaged must be selected before the pillar extraction, precluding zooming capability. 


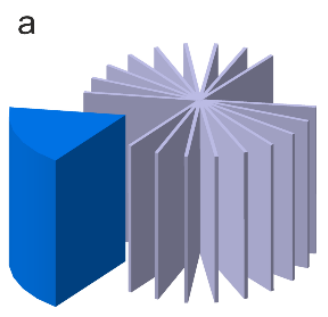

b
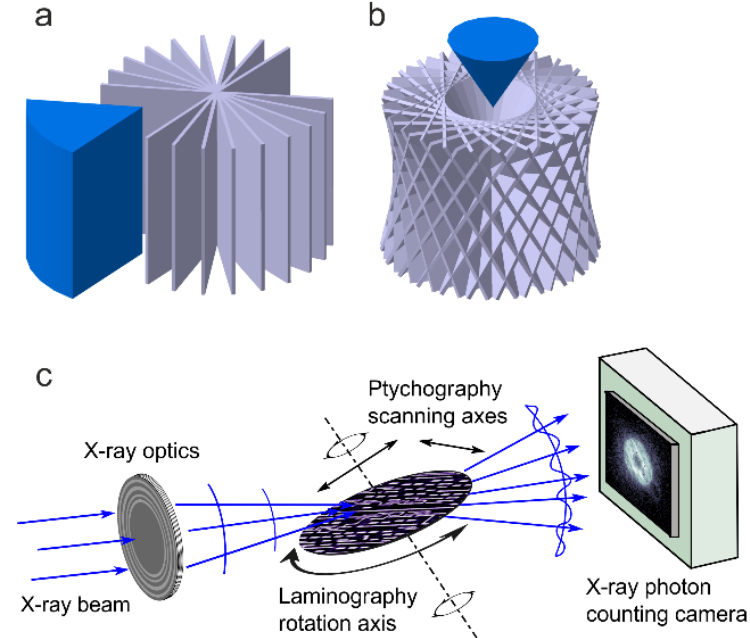

d

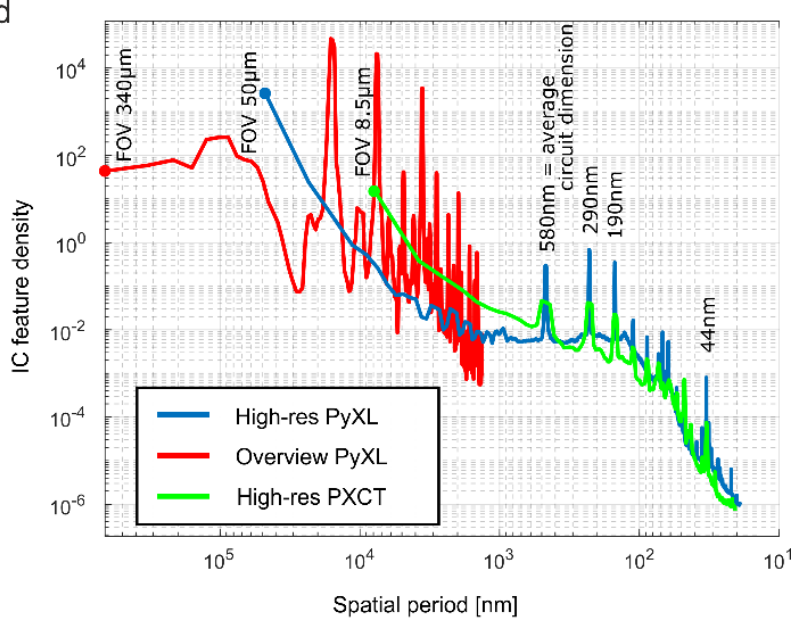

Fig. 1 Imaging geometries.

a, Illustration of the filling of Fourier space in tomography (missing wedge) for conventional tomography with $\Theta=90$ degrees and $\mathbf{b}$ laminography (missing cone) for $\Theta=61$ degrees as in our instrument. c, Imaging geometry of X-ray ptychographic laminography (PyXL). d, Density of chip features as a function of the in-plane component of the spatial period for PyXL in high resolution limited field of view (FOV) shown in blue and low resolution overview (with expanded FOV) modes shown in red, as well as for conventional PXCT depicted in green. The spikes are real and due to the multiscale design rules for the chip.

The preparation of cylindrical pillars from a region of interest could be avoided for tomography by mounting the sample with the axis of rotation parallel to the integrated circuit surface. However, this causes the effective thickness of the sample to increase in proportion with the incidence angle of the $\mathrm{X}$-rays to the sample. The increased effective thickness increases the attenuation and thereby limits the angular range of the measurement, resulting in a missing wedge of data in reciprocal space (Fig. 1a). The situation may be improved by not having the axis of rotation perpendicular to the incoming beam. Imaging in this generalized geometry is called laminography ${ }^{9,10,11}$ and hierarchical X-ray imaging has been demonstrated at low resolution ${ }^{12}$. The approach is well suited to flat samples, where the tilted rotation axis forbids access to momentum transfers within a cone - rather than wedge - of reciprocal space (Fig. 1b).

While it may seem, from the point of view of missing information, that the two geometries are similar, laminography has several important advantages. First, because the angle between the incoming X-ray beam and the surface is constant, the effective sample thickness to penetrate remains constant as well. Second, laminography simplifies preparation of planar samples to at most a uniform thinning of the substrate to manage attenuation, and provides flexibility to select the measurement position and to trade, for a given photon budget, resolution for measured volume. More specifically, zooming can be achieved by combining a variety of straightforward measures, including decreasing the sample raster steps and X-ray spot size, and increasing the counting times to capture more reliable high-angle scattering events.

\section{Ptychographic X-ray laminography}

In this Article, we report the 3D imaging of planar samples using ptychographic X-ray laminography (PyXL) (Fig. 1c). Ptychography scans are performed by moving the sample in a plane perpendicular to the axis of rotation. Thereby the sample is a fixed distance from the beam conditioning optics, allowing the use of conventional ptychography algorithms. We developed and built an instrument dedicated to PyXL in which the sample position is measured with respect to beam defining optics via differential laser interferometry ${ }^{13}$, similar to the previously described tomography instruments ${ }^{14,15}$. The interferometer positions are used to operate a piezo scanner in closed-loop, which in turn is mounted on an axis of rotation for laminography. The diffracted X-rays propagate to a pixelated photon-counting detector in 
the far-field through a helium-filled flight tube. Choosing the laminography angle $\theta$ requires a balance between minimizing the missing information cone and providing sufficient transmission through the sample, and is in our case set to $\theta=61$ degrees. While the scan range of the piezoelectric stage is only $100 \times 100 \mu \mathrm{m}^{2}$, the axis of rotation is mounted on two linear stages allowing stitching of several such scan regions and thereby sampling any selected region of interest in the sample. The maximum sample area that can be imaged in the instrument is $12 \times 12 \mathrm{~mm}^{2}$, beyond the die size of many integrated circuits in daily use.

We also address the image reconstruction challenge created by the missing cone of information in reciprocal space (Fig. 1b), which is inherent to the laminography geometry (Fig. 1c). When standard reconstruction methods such as filtered backprojection ${ }^{16,17}$ are used, the missing cone remains empty, resulting in unphysical artifacts (Supplementary Fig. 1b). Instead, we use an iterative approach that enforces consistency in the Fourier (reciprocal space) domain between the reconstruction and the measured data and two additional priors in real space. The first prior is based on knowledge of the sample composition and limits the electron density of the sample material to a range between that of $\mathrm{SiO}_{2}$ and $\mathrm{Cu}$. The second prior uses the assumption of a compact shape of the copper interconnects by driving the solution towards a minimal local total variation ${ }^{18}$. Iterative enforcement of these constraints in combination with a pyramidal multiscale approach improves the 3D reconstruction considerably (Supplementary Fig. 1c). Note that the refined reconstruction is still fully consistent with all measured data. In the future, to have a more self-contained approach, it may be possible to learn the density constraints from the measured data and, in this way, eliminate introduction of ad-hoc priors.

\section{Imaging of integrated circuits and method comparison}

We demonstrate PyXL by imaging an integrated circuit and comparing the result to its Graphic Database System (GDS-II) photolithographic mask layout files and also to the more conventional imaging approaches of PXCT and scanning electron microscopy (SEM).

The samples used are several identical chips produced in $16 \mathrm{~nm}$ fin field-effect transistortechnology for general purpose logic. The transistors are on a single crystal silicon substrate and are electrically connected by more than a dozen metal interconnect layers that are embedded in $\mathrm{SiO}_{2}$ dielectric. The convention is to label the metal layers in sequence, $\mathrm{M0}, \mathrm{M} 1, \mathrm{M} 2$, and so on, with M0 closest to the transistor layer. The total thickness of all of the layers and interconnect vias is about $\mathrm{T}=4 \mu \mathrm{m}$. We define $\mathrm{T}$ as the active layer thickness. For PXCT, a cylindrical pillar of $10 \mu \mathrm{m}$ diameter was prepared using a focused gallium ion beam (FIB) in an SEM and measured as for previous studies ${ }^{5}$. In total, 914 projections were acquired and we obtained an isotropic resolution of $16.5 \mathrm{~nm}$, determined using Fourier shell correlation ${ }^{19}$. Further details are given in Methods.

For laminography, we mechanically polished an entire chip of $2.5 \times 2.5 \mathrm{~mm}^{2}$ to a substrate thickness of about $20 \mu \mathrm{m}$ to obtain sufficient transmission of approximately $25 \%$ for $6.2 \mathrm{keV}$ X-rays. Supplementary Fig. 2 shows a visible light micrograph of the chip surface with the circular flip-chip solder bonding areas clearly seen. These have a pitch of $170 \mu \mathrm{m}$. At first, to obtain an overview image covering the region of interest and also demonstrating the stitching capabilities of the instrument, a near-field ptychography scan $^{20}$ was performed with a $50 \mu \mathrm{m}$ X-ray beam diameter. A total area of $300 \times 300 \mu \mathrm{m}^{2}$ was covered by stitching ptychographic measurements from 25 sub-scans, each containing a region of $70 \times 70 \mu \mathrm{m}^{2}$. In this overview mode the resolution obtained was $0.5 \mu \mathrm{m}$, limited by the reconstruction pixel size, which is given by the beam numerical aperture and the detector pixel size. Further details can be found in Methods. A low resolution 3D PyXL overview image was reconstructed using 300 of such projections. For the high-resolution PyXL measurement a $40 \mu \mathrm{m}$ diameter region was virtually selected in the overview dataset, also containing the region measured via PXCT in the first sample. The X-ray beam size was reduced to $4 \mu \mathrm{m}$ and $\mathrm{N}=2872$ projections were recorded in an angular range from 0 to 360 degrees. With a chip active layer thickness of $\mathrm{T}=4 \mu \mathrm{m}$ this angular sampling would allow a resolution no better than $7.6 \mathrm{~nm}$ at a laminography angle $\Theta$ of 61 degrees according to the laminography sampling equation, $\Delta \mathrm{r}=\pi \frac{T}{N} \tan \theta$, derived in Methods. This is lower than the limit of $12 \mathrm{~nm}$ posed by depth of field. ${ }^{21}$ 


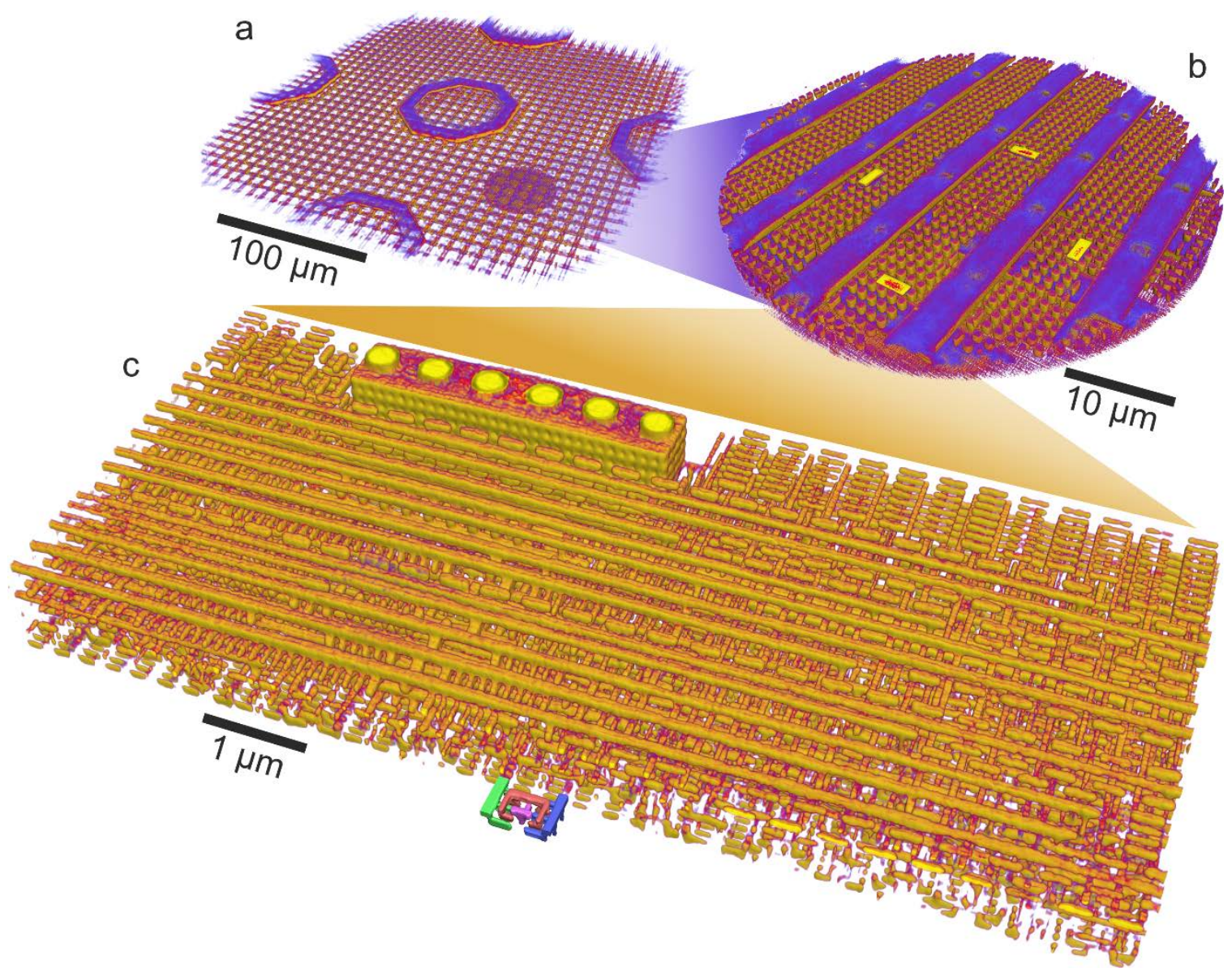

Fig. 2 3D renderings at different zoom levels covering a length-scale range from a $\mathbf{3 0 0} \boldsymbol{\mu m}$ overview to nanometric device structures.

The near-field ptychography results are shown in a giving a low resolution overview measurement covering a chip area of $300 \times 300 \mu \mathrm{m}^{2}$ with $500 \mathrm{~nm}$ resolution. The area measured via PyXL at high resolution is highlighted in a and shown in an isolated view in $\mathbf{b}$. Further zooming to the active structures is shown in c revealing fine details down to the reconstruction resolution of $18.9 \mathrm{~nm}$. All device layers and connections between them are resolved. The front edge of the volume rendering is cropped to expose the manually segmented inverter logic presented in Fig. $3 a$ in detail.

Figure $1 \mathrm{~d}$ shows the densities of chip features as a function of their spatial period, collected using PXCT and PyXL. It was computed in the two lowest layers referred to as MO and M1 by means of a normalized power spectral density of the reconstructed electron density. The sharp peaks are real and due to the chip's hierarchical design rules, which impose quasi-periodic features for periods varying by several orders of magnitude. Naturally the peaks are sharper (and more intense) for the larger fields of view possible for PyXL because the sampled volumes are larger, implying better definition of quasi-periodic structures, and so leading to two additional decades of useful spatial information in the form of visible maxima for PyXL compared to PXCT. The large advance represented by PyXL is thus very clear: with a single instrument we are now able to efficiently visualize the multiscale "connectome" for an integrated circuit from the millimetre to ten nanometre scales. Figure 2 shows 3D renderings of the reconstructed volumes measured via PyXL at various length scales down to the device level. The rendering was created by applying a threshold to the data and it covers five orders of magnitude in length scales, revealing device details as well as features at the $100 \mu \mathrm{m}$ scale of the overview. Supplementary movie 1 shows such zooming capabilities in an animated way, and Supplementary Fig. 3 shows slices of the raw reconstructions of the two lowest metal layers of the chip, $\mathrm{M} 0$ and $\mathrm{M} 1$, unaltered by thresholding and segmentation. 


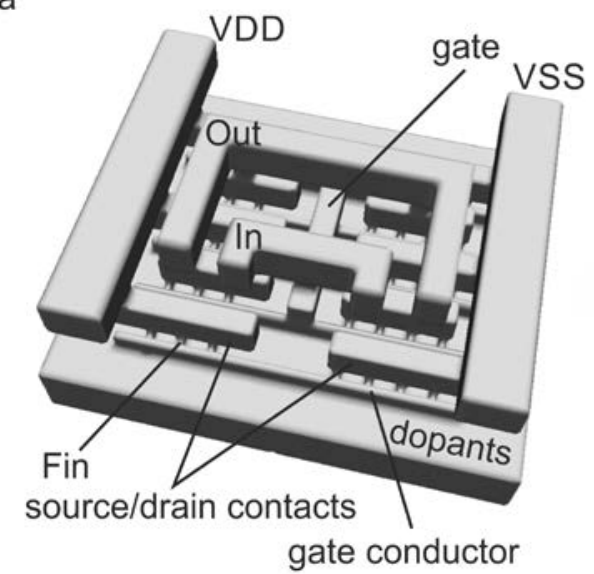

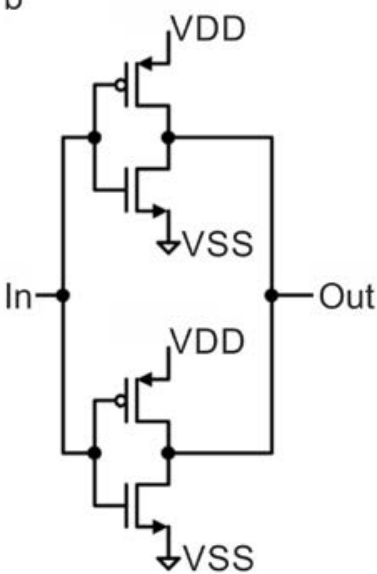

C

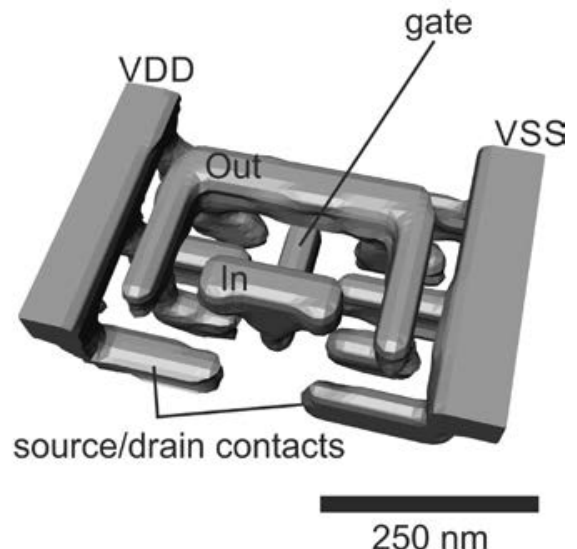

Fig. 3. 3D rendering and circuit diagram of an inverter.

VDD and VSS are supply voltages of the device, "In" labels the input and "Out" the output. The associated metal layer is M1. The expected device structure generated from the GDS-II model is rendered in a. Here also details not resolved in the measurement are shown, such as the Fins and gate conductors. The electronic circuit representation is shown in $\mathbf{b}$. The structure identified in the volume of the high-resolution PyXL measurement is depicted in c. It has been manually segmented and is shown as an interpolated surface rendering. The $250 \mathrm{~nm}$ scale bar is valid for $\mathbf{a}$ and $\mathbf{c}$.

For both PXCT and PyXL, the resolution is sufficient to identify all interconnections down to the individual transistors, even though the laminography resolution volume is anisotropic because of the missing cone of information (Fig. 1b). For tomography, Fourier shell correlation ${ }^{19}$ is an established method for estimating the resolution. In the case of laminography it remains a valid estimator as long as it is only computed in the valid Fourier regions and not in the missing cone. Such analysis will provide a measure of the obtained average resolution in the Fourier directions where data are available, meaning the resolution will worsen along the direction of the rotation axis. For the present measurement a resolution of $18.9 \mathrm{~nm}$ was estimated and thus we expect that imaging of even more advanced technology is possible today. As an example, on passing from $16 \mathrm{~nm}$ to $7 \mathrm{~nm}$ technology, the minimum metal pitch in technology decreases from $64 \mathrm{~nm}$ to $40 \mathrm{~nm}$, still considerably larger than our present resolution. We did not procure a physical chip paired with a design file at the $7 \mathrm{~nm}$ node, so for this first demonstration of PyXL, $16 \mathrm{~nm}$ technology was imaged instead.

An interesting question, especially in the context of security, is whether and where certain electronic building blocks of a particular type exist within a manufactured integrated circuit. As an example, an inverter circuit was chosen and to automate the search, we used the known GDS-II model to render a corresponding 3D volume with a voxel size identical to the PyXL reconstruction, shown in Fig. 3a next to Fig. 3b, which is the underlying electrical circuit schematic. We then calculated the expected reconstruction volume by removing its Fourier components within the missing cone in reciprocal space. The coordinates of the inverter pattern, corresponding to the GDS-II model, were then found in the measured dataset at low computational cost using 3D cross-correlation. We manually segmented the single inverter found in the PyXL dataset and rendered it as the interpolated surface view shown in Fig. $2 \mathrm{c}$ and 3c. The expected structure rendered from the GDS-II file in Fig. 3a shows also individual Fins that cannot be identified in the measured data. Supplementary movie 2 shows the slices of the inverter (Fig. 3) with the measured image and GDS-II model side by side. The automatic search of circuitry from the GDS-II in the measured data can be extended to larger blocks and thereby provides a fast method to verify that chip design and manufacture match and no undesired functionality (e.g. a backdoor into a processor or memory) was introduced.

PyXL represents the only non-destructive method for nanometer-resolution analysis of integrated circuits. Commonly used methods, such as mechanical delayering and imaging by SEM, for nanometerscale imaging of integrated circuits are destructive. As a consequence not only can the sample not be re-examined after the imaging process, but also the images obtained may not even reflect the prior 
state of the IC because of sample modification during the preparation steps. The non-destructive nature of PyXL therefore may be especially useful when investigating IC failure, as caused for example by electrostatic discharge. Nonetheless, it remains important to compare the imaging quality of PyXL with established techniques, for which one of our samples was mechanically polished to expose the second lowest metal layer, referred to as M1, and imaged in an SEM with electron energy of $5 \mathrm{keV}$. Figure 4 shows the direct comparison of the results obtained by SEM, PXCT and PyXL. For completeness, the corresponding (simplified) photolithographic mask layout of the same layer is also shown. Clearly, SEM provides highest image quality within a given 2D plane. However, it is a destructive multi-step technique and artifacts may occur due to the unavoidable and imperfect mechanical removal of material to expose the sample plane of interest. For example, the failure to mechanically delayer to the precise location of the M1 metal layer is apparent because bright spots in the image correspond to the location of vias connecting M1 to the third lowest layer M2.
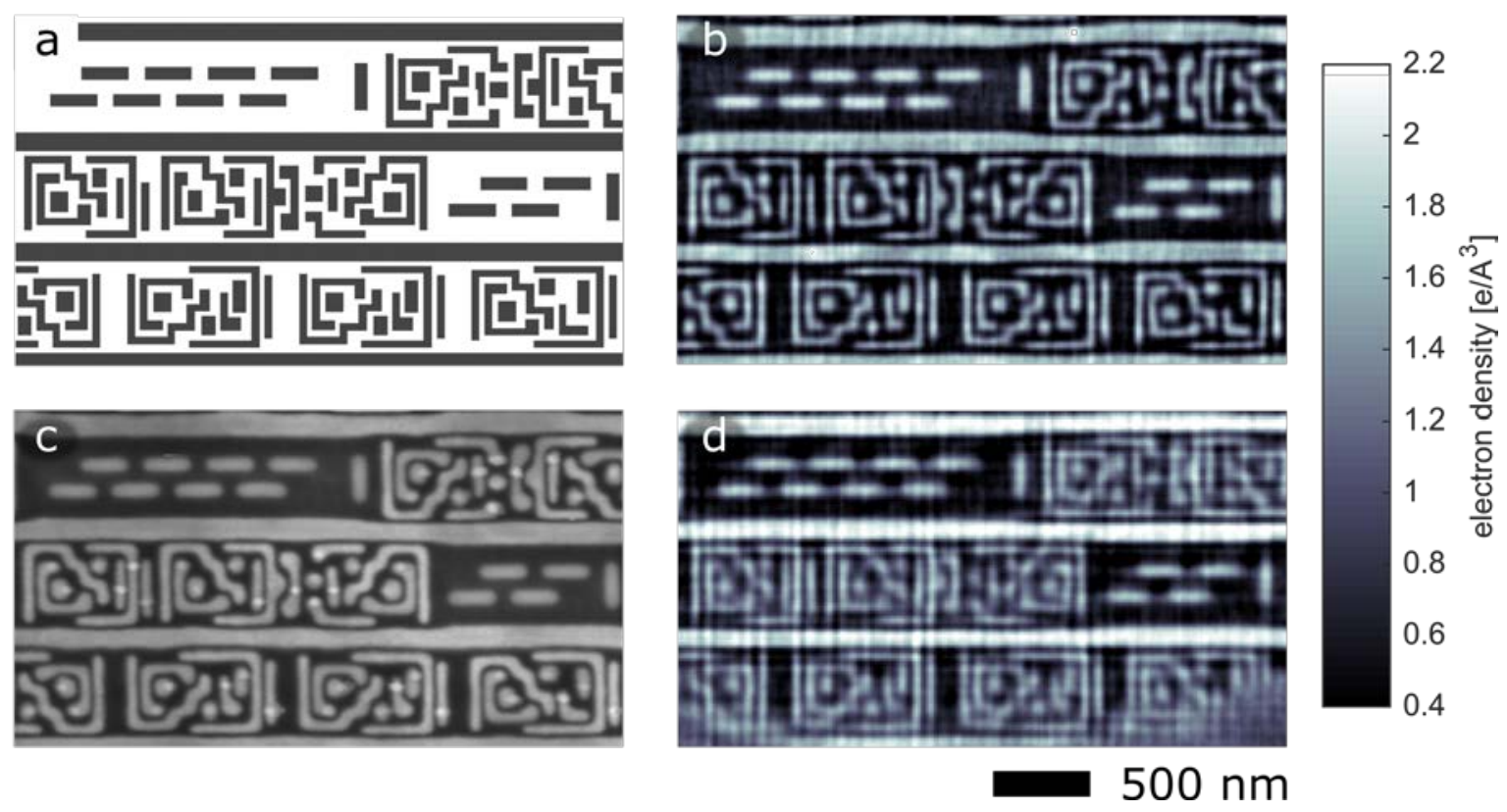

Fig. 4 Comparison of different imaging techniques applied to different samples of the same chip area, showing the second lowest metal layer M1.

a, Design layout (simplified), virtual slices obtained from $\mathbf{b}$ ptychographic $X$ ray computed laminography (PyXL) after missing cone refinement, c scanning electron microscopy (SEM), d ptychographic X-ray computed tomography (PXCT). The gray scale bar shown applies to the quantitative $X$-ray images in $\mathbf{b}$ and $\mathbf{d}$, and the bright lines correspond to connections manufactured from $\mathrm{Cu}$ surrounded by $\mathrm{SiO}_{2}$. The region of interest shown here was close to the edge of the extracted pillar for PXCT in $\mathbf{d}$, which explains the missing features in the lower right corner.

PyXL is well suited for imaging integrated circuits, and in-plane features provided by PyXL are clearer and contain fewer artifacts compared to PXCT. This is most likely due to a combination of 1) a limited depth of field ${ }^{21}$ which is less limiting for PyXL because in this measurement geometry the effective thickness along the $X$-ray propagation is slightly smaller, and 2) because of the improved nanostructural stability of a wafer (PyXL) relative to that of an isolated pillar (PXCT) when strongly irradiated by $X-$ rays $^{5}$. The streaking artifacts for PXCT are caused by the long and straight metal line features, which dominate when imaged parallel to the direction of $X$-ray propagation resulting in phase jumps larger than $2 \pi$, and thereby causing artifacts in the reconstruction. For PyXL the X-rays are not parallel to the direction of these structures, making PyXL less prone to these artifacts. However, for PyXL the resolution is anisotropic which results in artifacts of a different type in the direction of the missing cone, i.e. perpendicular to the individual layers. For comparison of PXCT and PyXL Supplementary Fig. 1 shows two slices perpendicular to the layers of the chip. Here the structures appear less sharp in the vertical direction for PyXL. 


\section{Conclusions}

We have reported PyXL, a technique that can image extended flat samples in $3 \mathrm{D}$ with nanoscale resolution and minimal sample preparation. Low resolution, fast 3D overview scans can be performed without any modification in the setup, and the region of interest for high resolution 3D imaging can be freely chosen based on the overview. For our test chip, such a low resolution ( $500 \mathrm{~nm})$ scan over an area of $300 \times 300 \mu \mathrm{m}^{2}$, yielding an image with $2.3 \times 10^{7}$ voxels (cubic with side length $500 \mathrm{~nm}$ ), was collected in 30 hours; a high resolution $(18.9 \mathrm{~nm})$ zoom over a sub-area $40 \mu \mathrm{m}$ in diameter, which produced $3800 \times 3800 \times 600$ voxels with dimensions $13 \times 13 \times 13 \mathrm{~nm}^{3}$, required 60 hours. The latter chip area was 16 times larger than that which we could image using conventional ptychographic X-ray tomography at $16.5 \mathrm{~nm}$ resolution in 23 hours, demonstrating that for the layered integrated circuit in the present case, our laminography measurement, where (unlike PXCT) the more feature-rich planar structures are projected onto both dimensions of the area detector, is about 6 times more efficient for generating significant information at high resolution. This includes the images themselves and also the possibility of finding functional features, such as the presence and location of a single inverter circuit.

Currently the imaging rate, volume and resolution are limited by the available coherent X-ray flux. As outlined previously ${ }^{5}$, we expect drastic improvements in the near future. Such improvements follow the transition from third to fourth generation synchrotron sources, as pioneered by the Swedish MAXLAB ${ }^{22}$, from which we expect an increase by two orders of magnitude in brilliance, and the construction of $\mathrm{MHz}$ repetition rate X-ray free electron lasers including LCLS II at SLAC in California. In addition, the spectral bandwidth used in our experiment, which is determined by a Si(111) monochromator, could be relaxed by an order of magnitude by using multilayer mirrors ${ }^{23}$. Furthermore, our FZPs provide limited efficiency compared to, for example, Kirkpatrick-Baez mirror systems ${ }^{24}$, which could provide an additional order of magnitude improvement. The total increase in coherent flux could thus reach $10^{4}$, which could be combined with further advances from using an evacuated flight tube and in-vacuum detection, implying even greater efficiency for PyXL.

The scaling laws ${ }^{25}$ for ptychographic imaging would thus allow approaching a resolution of $2 \mathrm{~nm}$ with the same measurement time as for the current experiment, or inspection of an entire $0.3 \times 0.3 \mathrm{~mm}^{2} \mathrm{IC}$ at $50 \mathrm{~nm}$ resolution in three hours. Beyond higher flux, reaching such imaging speed and/or resolution will require additional developments for faster and more accurate sample scanning, improved X-ray probe stability and a careful management of radiation damage. In that context, the latest mechanical and electronic hardware can already improve the imaging rate by a further decrease in imaging overhead ${ }^{26}$. Additionally, laminography reconstruction algorithms could benefit from the sparse, laminar structure of ICs, as well as knowledge of other priors from design files and fabrication practice, leading to measurements requiring fewer projections. In the area of ptychographic reconstruction significant development is necessary and ongoing ${ }^{21,27,28}$ in order to reliably overcome the depth of field limitation, $12 \mathrm{~nm}$ in the present sample, to approach $2 \mathrm{~nm}$. Radiation damage may pose another limit to achievable resolution, although as mentioned above we observe significantly better sample stability for laminography compared to tomography requiring isolated pillars. Small deformations that may occur during a measurement at higher dose can also be dealt with in the reconstruction step ${ }^{29}$.

Beyond the measurement of integrated circuits for failure analysis, design validation and quality control, our imaging method is applicable to any scientific, medical or engineering problem where the samples are planar. In reflection mode, its application could become a practical 3D imaging technique for unthinned samples, further opening up low energy (soft) X-ray investigations in areas from biomedicine to magnetic devices.

\section{Methods}

\section{X-ray measurements}

All X-ray measurements were carried out at the cSAXS beamline of the Swiss Light Source (SLS) at the Paul Scherrer Institut (PSI), Switzerland. The photon energy of $6.2 \mathrm{keV}$ with a spectral bandwidth of $2 \times 10^{-4}$ was selected by a double crystal silicon monochromator. For both PXCT and PyXL a $170 \mu \mathrm{m}$ diameter gold Fresnel zone plate (FZP) with $60 \mathrm{~nm}$ outermost zone width was used in combination with a $50 \mu \mathrm{m}$ central stop and $30 \mu \mathrm{m}$ order sorting aperture to define a 3-4 $\mu \mathrm{m}$ diameter illumination on the sample. The FZPs were manufactured in the Laboratory for Micro and Nanotechnology (LMN), Paul Scherrer Institut, Switzerland based on a nanofabrication process published elsewhere ${ }^{30}$. 
The PXCT data was measured using the flexible tomography nano-imaging (flOMNI) instrument ${ }^{13,14,31}$ with a Pilatus photon counting detector ${ }^{32}$ with $172 \mu \mathrm{m}$ pixel size positioned at a distance of $7386 \mathrm{~mm}$ from the sample, using $600 \times 600$ pixels for the reconstruction resulting in a reconstructed pixel size of $14.3 \mathrm{~nm} .914$ projections were acquired, each having a field of view of $18 \times 9 \mu \mathrm{m}^{2}$. The $328 \mathrm{scan}$ positions of each projection followed a Fermat spiral trajectory ${ }^{33}$, had an average spacing of $0.7 \mu \mathrm{m}$, and $0.1 \mathrm{~s}$ exposure time. The sample was positioned downstream from the focal point of the FZP where the beam diameter was $3 \mu \mathrm{m}$. The measurement time per projection was 90 seconds. The deposited X-ray dose into the copper interconnects for the whole tomogram was estimated to be $55 \mathrm{MGy}$. The tomogram was reconstructed to a voxel size of $14.3 \mathrm{~nm}$ akin to the procedure used in Ref. 5: the complex-valued projections were reconstructed by 1200 iterations of the difference map solver ${ }^{34}$ followed by 300 iterations of the maximum likelihood refinement ${ }^{35}$. For tomography, the phase of the reconstructed projections was used after post-processing alignment and removal of constant and linear phase components ${ }^{36}$. A modified filtered back projection was used after aligning the projections using a tomographic consistency approach ${ }^{37}$.

PyXL laminography measurements were carried out using the laminography nano-imaging (LamNI) instrument. For the high-resolution scan, a circular field of view (FOV) of $40 \mu \mathrm{m}$ diameter was virtually defined in the sample surface plane such that the imaged volume is about a factor 16 larger compared to the case of PXCT. Because of the device geometry and the position measurement being perpendicular to the $\mathrm{X}$-ray beam propagation direction, this circular FOV in the sample plane results in an elliptical FOV of about $50 \times 26 \mu \mathrm{m}^{2}$ in the position-measurement plane, which is perpendicular to the $X$-ray beam propagation direction, as shown in Supplementary Fig. 4. The 485 scan points in that region of interest followed the Fermat spiral trajectory ${ }^{33}$, had an average spacing of $0.7 \mu \mathrm{m}$ in the positionmeasurement plane, and $0.1 \mathrm{~s}$ exposure time. The sample was positioned downstream from the focal position where the beam diameter was $4 \mu \mathrm{m}$. 2872 projections were recorded at angles equally spaced in the range of 0 to 360 degrees. The time to record a single projection was $67 \mathrm{~s}$ and the overhead between two projections for rotating and following the region of interest was $9 \mathrm{~s}$. The resulting X-ray dose experienced copper interconnects for the whole laminogram is estimated at 76 MGy. The complex-valued projections were reconstructed by 300 iterations of the difference map solver ${ }^{34}$ followed by 200 iterations of the maximum likelihood refinement ${ }^{35}$ using $480 \times 480$ pixels of the Eiger photoncounting detector ${ }^{38}$ with $75 \mu \mathrm{m}$ pixel size positioned at a distance of $2345 \mathrm{~mm}$ after the sample resulting in a pixel size of $13.0 \mathrm{~nm}$. Supplementary Fig. $4 \mathrm{~b}$ shows a reconstructed projection. The phase projections were unwrapped and aligned using a new in-house developed iterative algorithm based on $3 \mathrm{D}$ consistency and a pyramidal approach in which the reconstruction and alignment are refined starting from low- to progressively increased resolution. The initial 3D volume reconstruction was provided by the filtered backprojection (FBP) method with the filtering kernel multiplied by $\sin (\Theta)$ to account for the laminography geometry and $\Theta$ being the laminography angle ${ }^{39}$. Given sufficient angular sampling, the FBP method provides an optimal reconstruction, $X_{F B P}$, up to the missing cone region in the Fourier space. The values inside the missing cone were estimated by an iterative scheme in which the 3D volume was Fourier transformed back and forth between object and reciprocal space. In reciprocal space, consistency with the known Fourier components, which were obtained in the previous step, was enforced outside of the missing cone. Additional prior knowledge about the sample, as discussed in the main text, was enforced in object space. This process can be described as an alternating projection method between two constraints, $\Pi_{o}$ that applies object space priors and $\Pi_{F}$ that enforces consistency in the Fourier space

$$
\begin{aligned}
& \Pi_{o}: X \rightarrow X_{o}=\max \left\{X_{\min }, \min \left[X_{\max }, X-\mu \nabla_{X} O_{T V}\right]\right\} \\
& \Pi_{F}: X \rightarrow X_{F}=X_{F B P}+\mathcal{F}^{-1}\left[S_{C} \mathcal{F}\left(X-X_{F B P}\right)\right]
\end{aligned}
$$

where $X$ denote the refined electron density and $X_{\min }, X_{\max }$ are limits of the maximum and minimum electron density values given by known composition of the sample. In object space, the refined reconstruction $X$ is additionally updated by a gradient of a regularization functional $\nabla_{X} O_{T V}$, in our case the total variation ${ }^{18}$, with updated step size $\mu$. Finally, in reciprocal space, i.e. after the Fourier transform $\mathcal{F}, S_{c}$ denotes the missing cone region defined as

$$
S_{c}\left(k_{x}, k_{y}, k_{z}\right)= \begin{cases}0, & \text { if } k_{x}^{2}+k_{y}^{2}<k_{z}^{2} \tan ^{2} \Theta \\ 1, & \text { else }\end{cases}
$$

where $k_{z}$ is the reciprocal space coordinate parallel to the rotation axis and $k_{x}, k_{y}$ are the perpendicular direction coordinates. An alternative approach would be an iterative use of the Radon transform to enforce consistency with the measured projections ${ }^{40}$, however the large volume of our reconstruction makes the Fourier transform-based approach significantly faster. Additionally, in order to further accelerate the convergence and reduce the computational cost, we have used a pyramidal approach, 
in which the missing cone refinement starts from the lowest spatial frequencies and progressively includes a larger fraction of the Fourier space while continuously enforcing the priors in the object space.

The reconstructed voxel size was $13.0 \mathrm{~nm}$ and the volume covers $3800 \times 3800 \times 600$ elements. The resolution was estimated to $18.9 \mathrm{~nm}$ using an intersection of the Fourier shell correlation (FSC) curve and the 1-bit threshold ${ }^{19}$. The missing cone was excluded from the FSC calculation. The computation of the 2D ptychographic reconstructions of this high-resolution laminography dataset required 4.7 hours using 8 nodes equipped with 28-core CPU Intel Xeon E5-2690 v4 processors. The computation time for the 3D laminography reconstruction using the filtered back projection method was 40 minutes using a single GPU Nvidia P100. The total time for the 3D reconstruction including data loading, alignment of the projections, filtered back projection reconstruction and the filling of the missing cone was 4 hours.

The near-field ptychographic laminography data shown in Fig. 2a and Supplementary Fig. 5b were measured using the identical setup as used for the far-field ptychographic laminography. The only required change of the beam diameter to $50 \mu \mathrm{m}$ was achieved by changing to a FZP with a diameter of $120 \mu \mathrm{m}$ instead of $170 \mu \mathrm{m}$, which we manufactured on the same membrane such that no manual interaction with the setup was required. The distance from the sample to the detector of $2345 \mathrm{~mm}$ and the Eiger detector pixel size of $75 \mu \mathrm{m}$ resulted in a projected pixel size on the image plane of $495 \mathrm{~nm}$, which was found to be the limit for the resolution. For this coarse laminogram, a field of view of $300 \times 300 \mu \mathrm{m}^{2}$ was measured with the Fermat spiral trajectory ${ }^{33}$ implemented with an average step size of $10 \mu \mathrm{m}$. To cover the field of view for each projection, 25 patches, each $70 \times 70 \mu \mathrm{m}^{2}$ in size, have been measured via stitching and reconstructed together. 300 projections were acquired, aligned using the same algorithms as for the high-resolution PyXL reconstruction. The time to measure each projection patch was $14 \mathrm{~s}$ including the overhead for moving to the next stitching patch with the coarse stage. Supplementary Table 1 gives a brief overview and comparison of the three X-ray measurements performed.

\section{Angular sampling requirements for laminography}

Similar to the Crowther criterion ${ }^{41}$ for conventional tomography, for laminography the angular step should be sufficiently small for adequate sampling of the object's 3D Fourier transform. Supplementary Fig. 6 a shows schematically the experimental realization with a fixed source under parallel beam, and Supplementary Fig. $6 \mathrm{~b}$ the equivalent representation with a fixed object and source rotating around it. Invoking the Fourier projection-slice theorem we know that each laminography projection contains information about a thin slice in the Fourier domain going through the origin and with normal parallel to the beam propagation direction, as shown schematically in Supplementary Fig. 6c, where the Fourier domain radius is inversely proportional to the real-space projection resolution $\Delta r$. The sampling requirement for laminography is that these Fourier slices are taken close enough to each other to capture all details in Fourier space along the direction parallel to the rotation axis, as illustrated in the inset of Supplementary Fig. 6c. Assuming that the sample thickness along the axis of rotation T is much smaller than the other sample dimensions, from this criterion it readily follows that

$$
\tan \theta=\frac{2 \Delta \mathrm{r}}{\mathrm{T} \Delta \varphi}
$$

where $\theta$ is the angle between the beam propagation direction and the axis of rotation, and $\Delta \varphi$ is the laminography angular step. Considering that for laminography the rotation is from 0 to $2 \pi$ the required number of projections $\mathrm{N}$ is given by

$$
\mathrm{N}=\frac{2 \pi}{\Delta \varphi}=\pi \frac{\mathrm{T}}{\Delta \mathrm{r}} \tan \theta
$$

For someone familiar with the Crowther tomography criterion ${ }^{41}$, it may seem counterintuitive that the equation is completely independent of the field-of-view (FOV), however, this is actually sensible and expected. The key difference with conventional tomography, which depends on the horizontal sample diameter, comes from the inclination of the measured Fourier slices, as shown in Fig. 1b. Because of this inclination we have guaranteed adequate sampling in the plane perpendicular to the rotation axis. Another way to intuitively understand that this equation should be independent of the FOV, is to consider that once we measure laminography projections we could always artificially reduce this FOV in postprocessing by cropping them, and of course we would not expect that by cropping the FOV our angular sampling would be relaxed.

Finally, the $\tan \theta$ factor shows that the most relaxed angular requirements are for small values of $\theta$, however this is only because for small values of $\theta$ the $3 \mathrm{D}$ information in the reconstruction is heavily 
reduced, both because the missing cone information grows significantly and because the resolution in the direction of the axis of rotation $\Delta \mathrm{z}$ is worsened

$$
\Delta \mathrm{z}=\frac{\Delta \mathrm{r}}{\sin \theta}
$$

Through numerical simulations we have observed that $\theta>\pi / 4$, provides a significant reduction of missing cone artifacts, and that best image quality is obtained for larger values of $\theta$, even if somewhat angularly undersampled.

\section{Data availability}

The aligned laminography projections generated during the current study are available under the Creative Common license in the Zenodo repository http://doi.org/10.5281/zenodo.2657340 doi:10.5281/zenodo.2657340

\section{Acknowledgements}

The measurements were performed at the cSAXS beamline of the Swiss Light Source (SLS) at the Paul Scherrer Institut (PSI).

\section{Author Contributions}

Sample preparation was done by E.M., S.F., and A.F.J.L. The X-ray measurements were carried out by M.H., M.O., and M.G.-S. The PyXL endstation was developed by M.H., J. R. The X-ray data were analyzed and visualized by M.H, M.O. and M.G.-S. Specialized algorithms for alignment and processing of projections and laminography reconstruction were developed by M.O. The X-ray illumination lens was produced by M.L. and C.D. The X-ray detector was developed by G.T. The FIB/SEM data were collected by J.Z., W.U., and A.F.J.L. The manuscript was written by M.H., M.O., M.G.-S., O.B., A.F.J.L., G.A.

\section{Competing financial interests}

The authors declare no competing financial interests. 


\section{References}

1. Behrens, T.E.J., Sporns, O. Human connectomics. Curr Opin Neurobiol 2012, 22(1): 144-153.

2. Shahmoradian, S., et al. Three-Dimensional Imaging of Biological Tissue by Cryo X-Ray Ptychography. Sci Rep 2017, 7: 6291.

3. Servanton, G., et al. Advanced TEM Characterization for the Development of $28-14 \mathrm{~nm}$ nodes based on fully-depleted Silicon-on-Insulator Technology. J. Phys.: Conf. Ser. 471, 012026

4. Gignac, L.M., et al. High Energy BSE/SE/STEM Imaging of 8 um Thick Semiconductor Interconnects. Microscopy and Microanalysis 2014, 20(S3): 8-9.

5. Holler, M., et al. High-resolution non-destructive three-dimensional imaging of integrated circuits. Nature 2017, 543(7645): 402.

6. Orji, N.G., et al. Metrology for the next generation of semiconductor devices. Nature Electronics 2018, 1(10): 532-547.

7. Robertson, J., Riley, M. The Big Hack: How China Used a Tiny Chip to Infiltrate U.S. Companies. 2018 [cited]Available from: https://www.bloomberg.com/2018-the-big-hack

8. Pfeiffer, F. X-ray ptychography. Nature Photonics 2018, 12(1): 9-17.

9. Hasenkamp, F.A. RADIOGRAPHIC LAMINOGRAPHY. Mater Eval 1974, 32(8): 169-+.

10. Helfen, L., et al. High-resolution three-dimensional imaging of flat objects by synchrotronradiation computed laminography. Applied Physics Letters 2005, 86(7): 071915.

11. Helfen, L., et al. Synchrotron-radiation computed laminography for high-resolution threedimensional imaging of flat devices. Phys Status Solidi A-Appl Mat 2007, 204(8): 2760-2765.

12. Xu, F., et al. Correlative Nanoscale 3D Imaging of Structure and Composition in Extended Objects. PLoS One 2012, 7(11): 6.

13. Holler, M., Raabe, J. Error motion compensating tracking interferometer for the position measurement of objects with rotational degree of freedom. Optical Engineering 2015, 54(5), 054101.

14. Holler, M., et al. An instrument for 3D x-ray nano-imaging. Review of Scientific Instruments 2012, 83(7): 073703.

15. Holler, M., et al. OMNY -A tOMography Nano crYo stage. Review of Scientific Instruments 2018, 89(4): 043706.

16. Myagotin, A., et al. Efficient Volume Reconstruction for Parallel-Beam Computed Laminography by Filtered Backprojection on Multi-Core Clusters. leee T Image Process 2013, 22(12): 5348-5361.

17. Kak, A.C., Slaney, M., Wang, G. Principles of Computerized Tomographic Imaging. Medical Physics 2002, 29(1): 107-107.

18. Chambolle, A. An algorithm for total variation minimization and applications. J Math Imaging Vis 2004, 20(1-2): 89-97.

19. van Heel, M., Schatz, M. Fourier shell correlation threshold criteria. J Struct Biol 2005, 151(3): 250-262.

20. Stockmar, M., et al. Near-field ptychography: phase retrieval for inline holography using a structured illumination. Sci Rep 2013, 3: 1927.

21. Tsai, E.H.R., et al. X-ray ptychography with extended depth of field. Optics Express 2016, 24(25): 29089-29108.

22. Eriksson, M., et al. Some small-emittance light-source lattices with multi-bend achromats. Nuclear Instruments \& Methods in Physics Research Section a-Accelerators Spectrometers Detectors and Associated Equipment 2008, 587(2-3): 221-226.

23. Bilderback, D.H., et al. Design of Doubly Focusing, Tunable (5-30 keV), Wide Bandpass Optics Made From Layered Synthetic Microstructures. Nuclear Instruments \& Methods in Physics Research 1983, 208(1-3): 251-261.

24. Kirkpatrick, P., Baez, A.V. Formation of Optical Images by X-rays. Journal of the Optical Society of America 1948, 38(9): 766-774.

25. Howells, M.R., et al. An assessment of the resolution limitation due to radiation-damage in X-ray diffraction microscopy. J Electron Spectrosc Relat Phenom 2009, 170(1-3): 4-12. 
26. Odstrcil, M., et al. Fast positioning for X-ray scanning microscopy by a combined motion of sample and beam-defining optics. J Synchrot Radiat 2019, 26(Pt 2): 504-509.

27. Suzuki, A., et al. High-Resolution Multislice X-Ray Ptychography of Extended Thick Objects. PRL 2014, 112(5): 053903.

28. Li, P., Maiden, A. Multi-slice ptychographic tomography. Sci Rep 2018, 8(1): 2049.

29. Odstrcil, M., et al. Ab initio nonrigid X-ray nanotomography. Nat Commun 2019, 10(1): 2600.

30. Gorelick, S., Guzenko, V.A., Vila-Comamala, J., David, C. Direct e-beam writing of dense and high aspect ratio nanostructures in thick layers of PMMA for electroplating. Nanotech 2010, 21(29), 295303.

31. Holler, M., et al. X-ray ptychographic computed tomography at $16 \mathrm{~nm}$ isotropic 3D resolution. Sci Rep 2014, 4: 3857.

32. Henrich, B., et al. PILATUS: A single photon counting pixel detector for X-ray applications. Nucl Instrum Methods Phys Res Sect A-Accel Spectrom Dect Assoc Equip 2009, 607(1): 247249.

33. Huang, X.J., et al. Optimization of overlap uniformness for ptychography. Optics Express 2014, 22(10): 12634-12644.

34. Thibault, P., et al. High-resolution scanning x-ray diffraction microscopy. Science 2008, 321(5887): 379-382.

35. Thibault, P., Guizar-Sicairos, M. Maximum-likelihood refinement for coherent diffractive imaging. N Jour Phys 2012, 14: 063004.

36. Guizar-Sicairos, M., et al. Phase tomography from x-ray coherent diffractive imaging projections. Optics Express 2011, 19(22): 21345-21357.

37. Guizar-Sicairos, M., et al. Quantitative interior x-ray nanotomography by a hybrid imaging technique. Optica 2015, 2(3): 259-266.

38. Guizar-Sicairos, M., et al. High-throughput ptychography using Eiger: scanning X-ray nanoimaging of extended regions. Optics Express 2014, 22(12): 14859-14870.

39. Helfen, L., et al. On the implementation of computed laminography using synchrotron radiation. Review of Scientific Instruments 2011, 82(6): 063702.

40. Harasse, S., Yashiro, W., Momose, A. Iterative reconstruction in x-ray computed laminography from differential phase measurements. Optics Express 2011, 19(17): 1656016573.

41. Crowther, R.A., DeRosier, D.J., Klug, A. The reconstruction of a three-dimensional structure from projections and its application to electron microscopy. Proceedings of the Royal Society of London A Mathematical and Physical Sciences 1970, 317(1530): 319. 


\section{Supplementary Information}
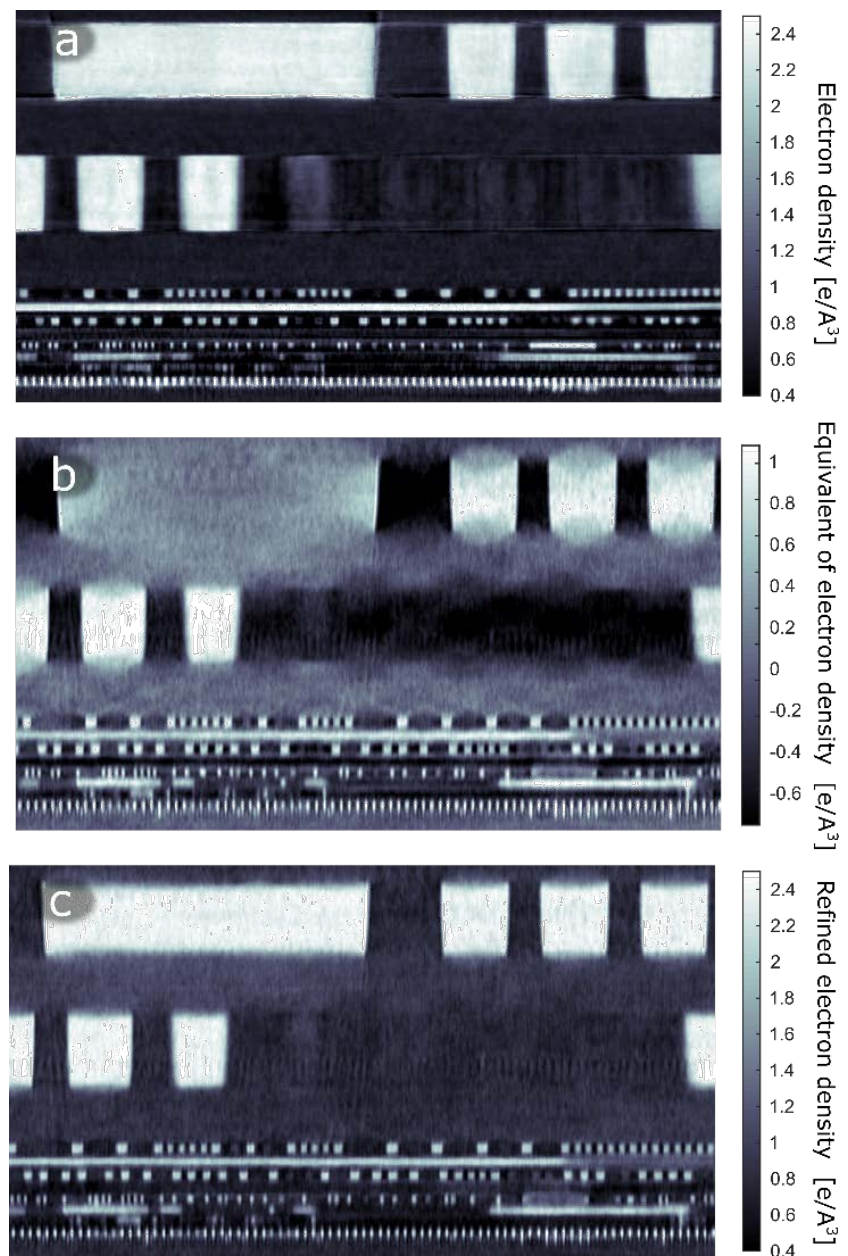

$2 \mu \mathrm{m}$

Supplementary Figure 1. Virtual coronal slices through the identical regions of the chip.

a measured by PXCT and $\mathbf{b}$ PYXL. In $\mathbf{c}$ the missing cone information was provided by a combination of material constraints and total variation minimization as described in the main text. Still it can be observed that the resolution in the vertical direction is worse compared to the horizontal direction as the missing information cannot be fully recovered in the case of PyXL. 


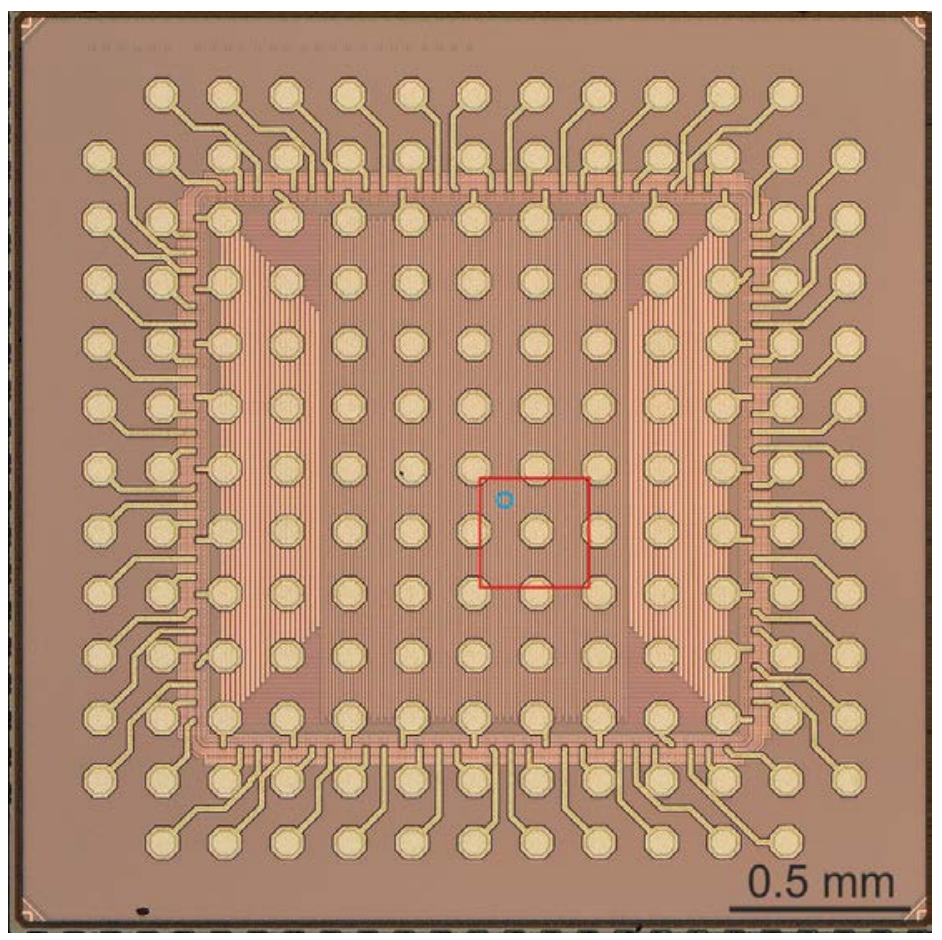

Supplementary Figure 2. Visible light microscopy image of the sample prepared for PyXL.

The chip is $2.5 \times 2.5 \mathrm{~mm}^{2}$ in area and the substrate was mechanically polished to a thickness of $20 \mu \mathrm{m}$. The red box indicates the area measured with PyXL at $500 \mathrm{~nm}$ resolution, and the blue circle indicates the area of the highresolution scan. 


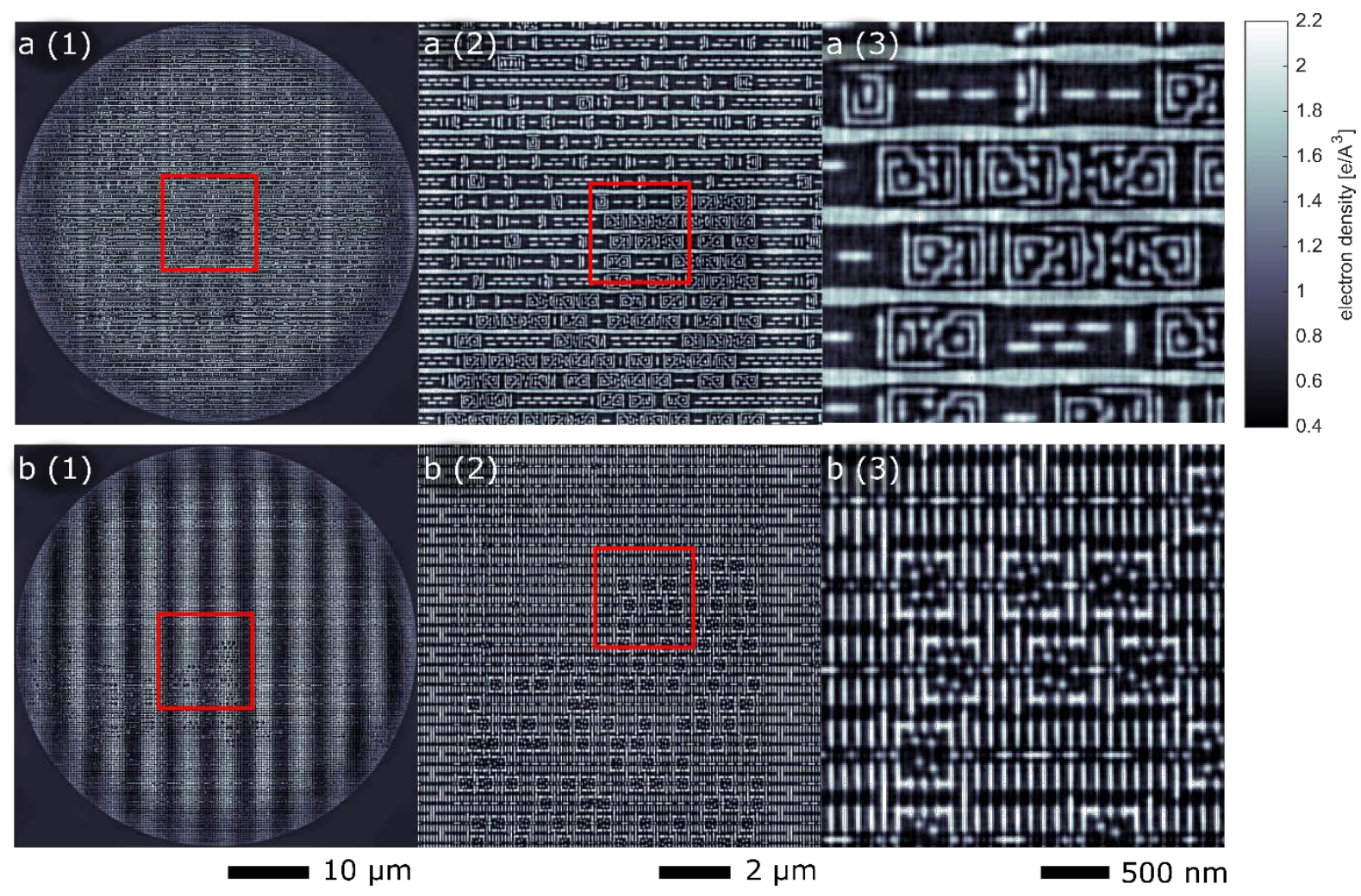

Supplementary Figure 3. Virtual slices through the two lowest metal layers. a shows layer M1 and $\mathbf{b}$ layer M0, of the dataset obtained by PyXL. The images on the right correspond to zoomed regions of the images to the left, where $a(1), b(1)$ show the full reconstruction FOV of about $50 \mu \mathrm{m}$ diameter. Towards the edge of the FOV the contrast drops because of reduced angular overlap. The actual measured field of view was $40 \mu \mathrm{m}$ in diameter. a (2) and $b$ (2) show an inset of $10 \times 10 \mu \mathrm{m}^{2}$, indicated as a square in a (1) and b (1). The smallest details can be seen in $a(3)$ and $b$ (3) which are $3 \times 3 \mu m^{2}$ insets of the area indicated by squares in $a(2)$ and $b(2)$, respectively.

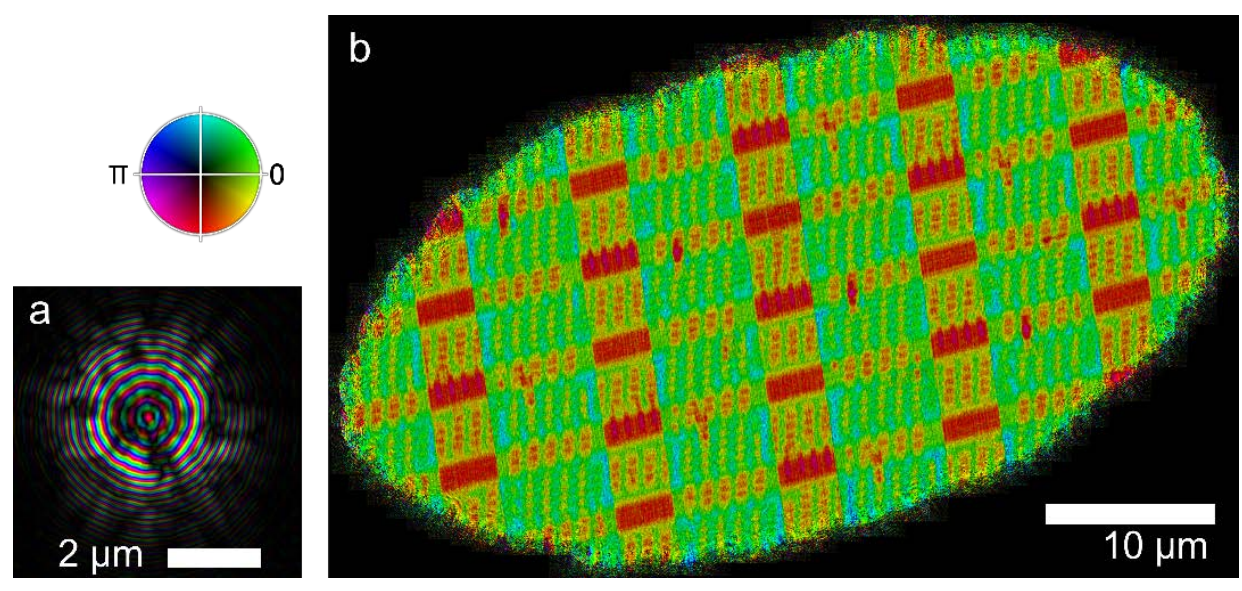

Supplementary Figure 4. Raw projection of the chip sample measured with PyXL.

a shows the reconstructed illumination probe and $\mathbf{b}$ the reconstructed projection of the sample, perpendicular to the beam propagation direction. The reconstructions are plotted in a complex color scale, where the brightness corresponds to amplitude and the hue indicates relative phase-shift. 


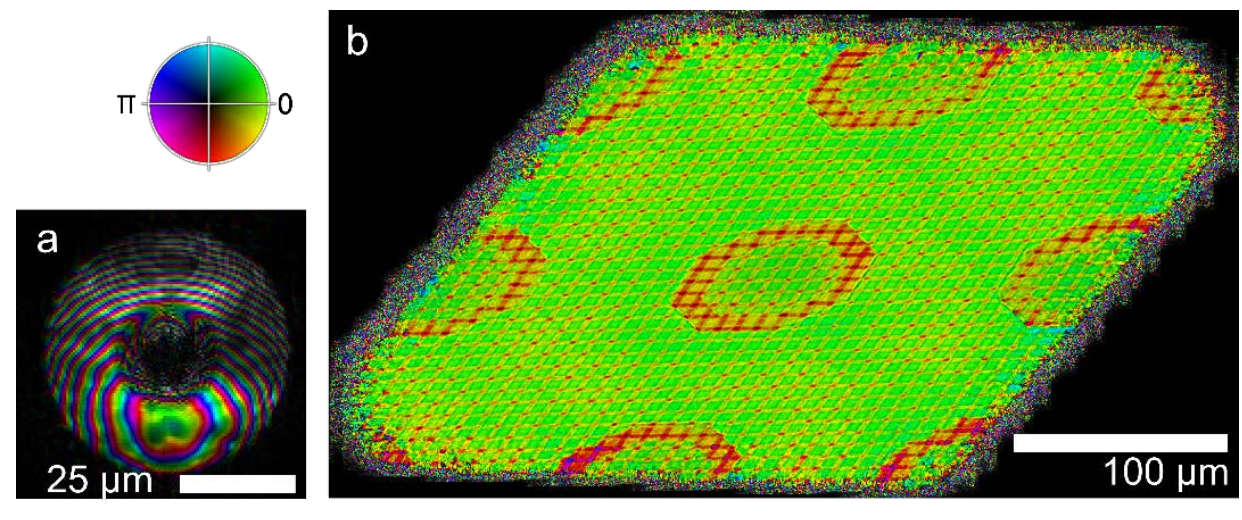

Supplementary Figure 5. A low resolution projection obtained from near-field ptychography with a $300 \times 300 \mu \mathrm{m}$ FOV.

$\mathbf{a}$ shows the reconstructed illumination probe and $\mathbf{b}$ a reconstruction projection of the sample. This low resolution scan was used for the 3D overview reconstruction shown in Fig. 2a. The reconstructions are plotted in a complex color scale, where the brightness corresponds to amplitude and the hue indicates relative phase-shift.
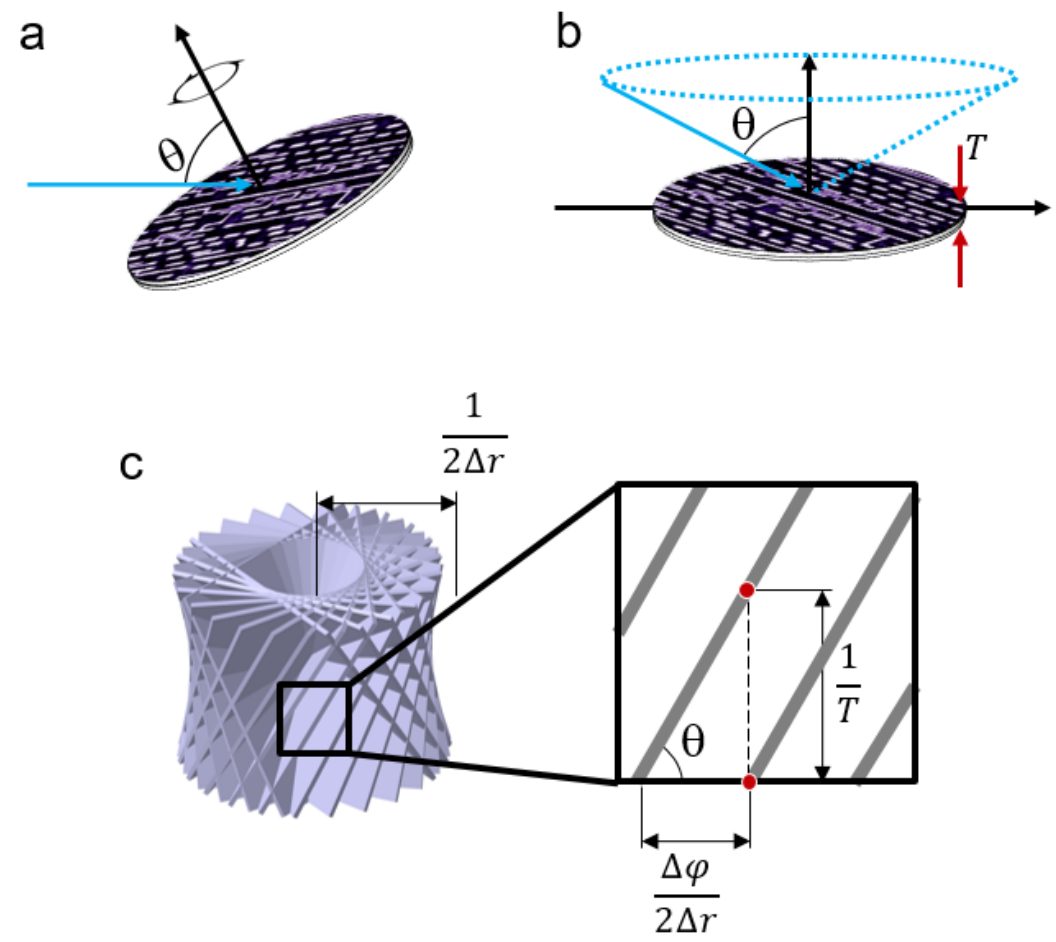

Supplementary Figure 6. Imaging geometry and angular sampling requirements.

a Laminography configuration with an angle $\theta$ between the beam propagation direction and the rotation axis. $\mathbf{b}$ Equivalent object-centric configuration where the beam propagation direction rotates around the object of thickness $T$. c Angular sampling requirements illustrated in Fourier domain. Each projection covers information over a thin slice in Fourier space. 
Supplementary Table 1. Overview of the parameters of the X-ray measurements

\begin{tabular}{|c|c|c|c|c|c|}
\hline & $\begin{array}{c}\text { sample } \\
\text { preparation }\end{array}$ & projection FOV & \# projections & $\begin{array}{l}\text { \# diffraction } \\
\text { patterns per } \\
\text { projection }\end{array}$ & $\begin{array}{l}\text { time per } \\
\text { projection }\end{array}$ \\
\hline PXCT & $\begin{array}{c}\text { FIB-SEM, } \\
10 \mu \text { diameter } \\
\text { pillar }\end{array}$ & $18 \times 9 \mu \mathrm{m}^{2}$ & 914 & 328 & $90 \mathrm{~s}$ \\
\hline $\begin{array}{c}\text { PyXL } \\
\text { overview }\end{array}$ & \multirow{2}{*}{$\begin{array}{c}\text { mechanical } \\
\text { polishing of } \\
\text { substrate to } 20 \\
\mu \mathrm{m} \text { thickness }\end{array}$} & $50 \times 26 \mu \mathrm{m}^{2}$ & 2872 & 485 & $67 \mathrm{~s}$ \\
\hline PyXL & & $\begin{array}{c}300 \times 300 \mu \mathrm{m}^{2} \\
\text { in } 25 \text { patches } \\
\text { each } \\
70 \times 70 \mu \mathrm{m}^{2}\end{array}$ & 300 & $25 \times 35$ & $25 \times 14 \mathrm{~s}$ \\
\hline
\end{tabular}

